\title{
Resting state magnetoencephalography functional connectivity in traumatic brain injury
}

\author{
Phiroz E. Tarapore, M.D. ${ }^{1}$, Anne M. Findlay, B.S., M.A. ${ }^{2}$, Sara C. LaHue, B.A. ${ }^{2}$, Hana Lee, \\ M.P.H. ${ }^{2}$, Susanne M. Honma, R.T. ${ }^{2}$, Danielle Mizuiri, B.S. ${ }^{2}$, Tracy L. Luks, Ph.D. ${ }^{2}$, Geoffrey \\ T. Manley, M.D., Ph.D. ${ }^{1}$, Srikantan S. Nagarajan, Ph.D. ${ }^{2}$, and Pratik Mukherjee, M.D., Ph.D. ${ }^{2}$ \\ ${ }^{1}$ Department of Neurological Surgery, University of California, San Francisco, California \\ ${ }^{2}$ Department of Radiology and Biomedical Imaging, University of California, San Francisco, \\ California
}

\section{Abstract}

Object-Traumatic brain injury (TBI) is one of the leading causes of morbidity worldwide. One mechanism by which blunt head trauma may disrupt normal cognition and behavior is through alteration of functional connectivity between brain regions. In this pilot study, the authors applied a rapid automated resting state magnetoencephalography (MEG) imaging technique suitable for routine clinical use to test the hypothesis that there is decreased functional connectivity in patients with TBI compared with matched controls, even in cases of mild TBI. Furthermore, they posit that these abnormal reductions in MEG functional connectivity can be detected even in TBI patients without specific evidence of traumatic lesions on 3-T MR images. Finally, they hypothesize that the reductions of functional connectivity can improve over time across serial MEG scans during recovery from TBI.

Methods-Magnetoencephalography maps of functional connectivity in the alpha (8- to $12-\mathrm{Hz}$ ) band from 21 patients who sustained a TBI were compared with those from 18 age- and sexmatched controls. Regions of altered functional connectivity in each patient were detected in automated fashion through atlas-based registration to the control database. The extent of reduced functional connectivity in the patient group was tested for correlations with clinical characteristics of the injury as well as with findings on 3-T MRI. Finally, the authors compared initial connectivity maps with 2-year follow-up functional connectivity in a subgroup of 5 patients with TBI.

Results-Fourteen male and 7 female patients (17-53 years old, median 29 years) were enrolled. By Glasgow Coma Scale (GCS) criteria, 11 patients had mild, 1 had moderate, and 3 had severe

\section{(c)AANS, 2013}

Address correspondence to: Pratik Mukherjee, M.D., Ph.D., Department of Radiology and Biomedical Imaging, University of California, San Francisco, California 94107-0946. pratik@ radiology.ucsf.edu.

Disclosure

Author contributions to the study and manuscript preparation include the following. Conception and design: Mukherjee, Manley, Nagarajan. Acquisition of data: Mukherjee, Findlay, LaHue, Lee, Honma, Mizuiri. Analysis and interpretation of data: Mukherjee, Tarapore, Findlay, LaHue, Luks, Nagarajan. Drafting the article: Mukherjee, Tarapore. Critically revising the article: all authors. Reviewed submitted version of manuscript: all authors. Approved the final version of the manuscript on behalf of all authors: Mukherjee. Statistical analysis: Tarapore. Study supervision: Mukherjee, Manley, Nagarajan. 
TBI, and 6 had no GCS score recorded. On 3-T MRI, 16 patients had abnormal findings attributable to the trauma and 5 had findings in the normal range. As a group, the patients with TBI had significantly lower functional connectivity than controls $(\mathrm{p}<0.01)$. Three of the 5 patients with normal findings on 3-T MRI showed regions of abnormally reduced MEG functional connectivity. No significant correlations were seen between extent of functional disconnection and injury severity or posttraumatic symptoms ( $p>0.05$ ). In the subgroup undergoing 2-year followup, the second MEG scan demonstrated a significantly lower percentage of voxels with decreased connectivity $(\mathrm{p}<0.05)$ than the initial MEG scan.

Conclusions-A rapid automated resting-state MEG imaging technique demonstrates abnormally decreased functional connectivity that may persist for years after TBI, including cases classified as "mild" by GCS criteria. Disrupted MEG connectivity can be detected even in some patients with normal findings on 3-T MRI. Analysis of follow-up MEG scans in a subgroup of patients shows that, over time, the abnormally reduced connectivity can improve, suggesting neuroplasticity during the recovery from TBI. Resting state MEG deserves further investigation as a prognostic and predictive biomarker for TBI.

\section{Keywords}

cognition; concussion; diffuse axonal injury; functional connectivity; magnetoencephalography; traumatic brain injury

Traumatic brain injury is a major cause of morbidity and disability worldwide. In the US, an estimated 1.7 million people sustain a TBI annually. ${ }^{8}$ Of these injuries, about $75 \%$ are concussions or other forms of mild TBI. ${ }^{25}$ Direct and indirect costs of TBI totaled an estimated $\$ 60$ billion in 2000 for the US alone. ${ }^{10}$ Despite the fact that nearly $80 \%$ patients with TBI are treated in an emergency department and released, long-term sequelae of even mild TBI such as persistent postconcussive syndrome can occur in up to $25 \%$ of patients. ${ }^{33}$ However, there are no current clinical criteria, conventional imaging methods, or laboratory tests that can reliably predict which patients with TBI will go on to have poor outcomes. Therefore, a better understanding of the pathophysiology of TBI is needed to develop prognostic biomarkers that can be used for patient counseling, selection for interventions such as cognitive rehabilitation and experimental trials of new pharmacological agents, as well as treatment monitoring in clinical research studies.

One mechanism by which TBI is thought to affect cognition and behavior is through alteration of functional connectivity. ${ }^{3,24}$ Functional connectivity refers to temporally correlated activity among spatially disparate brain regions and is indicative of neural communication between these regions. ${ }^{1,11,30}$ Because of the brain's interconnectedness, a single focal lesion can have deleterious effects not just locally, but also in remote, functionally connected sites. ${ }^{12,29,35}$ Conversely, in patients in whom no lesion is radiographically apparent, functional connectivity analysis can offer a means of quantifying a pathophysiological process that may otherwise only have clinical manifestations. ${ }^{16,21,22}$ Magnetoencephalography has been used for studying functional connectivity in diverse diseases including brain tumors, $, 26,12,23$ schizophrenia, ${ }^{14,15}$ and stroke. ${ }^{7,38}$ It is noninvasive, painless, and, because it measures magnetic fields, is not subject to the distortion introduced by variable conductance of tissues inherent to electroencephalography. 
This pilot study using a rapid automated MEG method suitable for routine clinical application aims to characterize the changes of resting state functional connectivity in patients with TBI compared with matched controls, including the longitudinal changes that occur during recovery from TBI. We collected 1-minute resting-state MEG recordings of neuromagnetic activity in the alpha frequency band $(8-12 \mathrm{~Hz})$ in this patient group and compared the results in automated fashion with resting state recordings taken from a group of age- and sex-matched controls. We chose to analyze the interregional connectivity of alpha waves because that is the frequency band with the greatest power during the resting state. ${ }^{12}$ We hypothesize that disruption of long-range communication due to focal cortical injury and shearing injury of long white matter tracts will cause a reduction of alpha-band MEG functional connectivity in TBI patients versus controls. We also postulate that MEG is particularly sensitive to brain injury and therefore can reveal decreases in functional connectivity even in the absence of radiographically evident focal lesions on 3-T MRI such as cortical contusions and microhemorrhages due to axonal injury. Finally, we hypothesize that functional recovery will be accompanied by increases in alpha-band MEG functional connectivity in serial MEG scans of a subgroup of TBI patients over a 2-year interval.

\section{Methods}

\section{Patient Population}

Patients were prospectively enrolled using the following inclusion criteria: age between 16 and 55 years of age who had suffered TBI within the last 4-30 months and with persistent self-reported symptoms. Exclusion criteria included history of neurological or psychiatric illness, including substance abuse, and contraindications to MRI including implanted devices, such as pacemakers, as well as pregnancy. We enrolled 21 patients with TBI of all severities. All patients underwent MEG and MRI at the University of California, San Francisco (Biomagnetic Imaging Laboratory). A subset of 5 patients also underwent a second MEG study 21-31 months after the initial MEG scan. Eighteen age-and educationmatched subjects were recruited for the control group and underwent the same MEG and MRI protocol as the patients. Control subjects were matched as a group to the patient cohort.

Demographic and clinical data pertaining to the mechanism of injury, initial imaging findings, and clinical course were obtained for each patient. All subjects gave written informed consent to participate in the research, and the study was conducted with Institutional Review Board approval.

\section{Magnetic Resonance Imaging}

All MRI studies were performed on a 3-T scanner (Excite, GE) using an 8-channel head coil. To provide anatomical head models for MEG analysis, a high-resolution 3D T1weighted whole-brain volume was acquired using a fast spoiled gradient-recalled echo in a steady-state inversion recovery (FSPGR-IR) sequence (TR $6.3 \mathrm{msec}$, TE $1.5 \mathrm{msec}$, TI 400 msec, and flip angle $15^{\circ}$ ), slice thickness $1.0 \mathrm{~mm}$, matrix size $256 \times 256$, and FOV $230 \times$ $230 \mathrm{~mm}$ with skin-to-skin coverage to include the nasion and preauricular points. 
Two additional 3-T MRI sequences to evaluate for the focal anatomical lesions of TBI included 1) axial T2-weighted FLAIR images (TE $126 \mathrm{msec}$, TR 10 seconds, and TI 2200 msec) with 220-mm FOV, 47-48 3.0-mm contiguous slices at a $256 \times 256$ matrix; and 2) axial magnetization prepared gradient echo (MPGR) T2*-weighted images (TE $15 \mathrm{msec}$, TR $500 \mathrm{msec}$, and flip angle $20^{\circ}$ ) with $220 \times 170-\mathrm{mm}$ FOV, and 47-48 3.0-mm contiguous slices at a $256 \times 192$ matrix.

All MR images were interpreted by a board-certified attending neuroradiologist experienced in the evaluation of brain MRI performed for head trauma.

\section{Magnetoencephalography Recordings}

Magnetic fields were recorded in a magnetically shielded room using a 275-channel wholehead CTF Omega 2000 system (VSM MedTech). A sampling rate of $600 \mathrm{~Hz}$ was used. Each individual lay on a comfortable bed. An artifact-free epoch of 1-minute duration out of 4 minutes of data collection, with the subject awake but resting quietly with his or her eyes closed, was selected for subsequent analysis.

\section{Signal Analysis Algorithms}

The MEG data obtained were bandpass filtered in the $8-$ to $12-\mathrm{Hz}$ alpha band. An adaptive spatial filtering algorithm was used to reconstruct the electromagnetic neural activity at each brain voxel from the signal recorded by the entire MEG sensor array. The details of this algorithm are described elsewhere. ${ }^{5,12,15,23,31,32,36}$ In brief, the raw MEG data were bandpass filtered with a fourth-order Butterworth filter, and the spatial covariance matrix was calculated from the entire recording of 1 minute in duration. We also computed the lead-field matrix, corresponding to the forward solution of a unit dipole at a particular location, for each voxel in the brain. From the spatial covariance and the forward field matrix, a spatial weight matrix was then obtained for optimal estimation of the signal power in each voxel. The activity at each time in each voxel was calculated as the linear combination of the spatial weighting matrix with the sensor data matrix. Thus, all sensors contributed in some degree to all voxel time series estimates from which we analyzed functional connectivity. Most of the commonly used measures of functional connectivity, such as coherence, ${ }^{4}$ phase locking value, ${ }^{18,19}$ and synchronization likelihood, ${ }^{34}$ overestimate the magnitude of true connectivity in this setting because of common references and crosstalk between voxels, a problem that is also well known in connectivity estimation from electroencephalography time series. ${ }^{9}$ In addition, traditional approaches to measuring functional connectivity are sensitive to volume conduction ${ }^{28}$ and spatial blur in reconstruction. Recently, an alternative method for estimating functional connectivity was introduced that overcomes the overestimation biases arising from crosstalk or volume conduction. ${ }^{26}$ This method, imaginary coherence, exploits the fact that phase similarities among time series arising from a common reference or from volume conduction occur with zero time delay. Thus, by omitting the mathematically real component of coherence, which mostly contains similarities with zero time lag, we remove suspect associations and limit the analysis to the mathematically imaginary component of coherence, which represents true interactions between brain areas occurring with a certain time lag. 


\section{Functional Connectivity Maps}

A 3D grid of voxels with an 8-mm spatial resolution covering the entire brain was created for each subject and recording, based on a multisphere head model of coregistered structural 3D T1-weighted FSPGR-IR MR images. Alignment of structural and functional images was ensured by marking 3 prominent anatomical points (nasion and preauricular points) on the subject's head in the MR images and localizing 3 fiducials attached to the same points before and after each MEG scan.

Magnetoencephalography oscillation frequencies between 1 and $20 \mathrm{~Hz}$ were used for calculation of the spatial weighting matrix and the voxel time series. For the calculation of IC, alpha frequency bins that showed the greatest power density during the resting state were chosen individually for each subject and averaged. A frequency resolution of $1.17 \mathrm{~Hz}(512$ frequency bins) was used. The connectivity, IC, at each voxel of interest was estimated by averaging across all its Fisher Z-transformed connections.

Maps of patient-specific connectivity changes (Pimages) were obtained by analyzing all pairwise connections between voxels of $2-\mathrm{cm}^{3}$ extension for each patient, resulting in approximately 30,000-60,000 voxel pairs in total depending on the individual's head size, and comparing to the mean of those obtained from all control subjects. From the point of view of a single voxel, the P-image reflects the mean imaginary coherence between that voxel and every other voxel in the brain. Thus a significant alteration in connectivity of that voxel means that its connectivity to the rest of the brain has changed. This alteration does not necessarily have implications for any specific other brain region. The computation time of the maps was reduced to about 10 minutes by distributing the processing of batches of voxel pairs to a cluster of 10 Linux workstations.

To test P-images of all 21 patients with TBI for differences with healthy control subjects, we spatially normalized them to the Montreal Neurological Institute atlas template according to the coregistered structural MRI, using the toolbox Statistical Parametric Mapping 8 (SPM8) for MATLAB (http://www.fil.ion.ucl.ac.uk/spm/software/spm8/). All normalized P-image voxels of TBI patients with Z-transformed connectivity estimates greater than or less than the $95 \%$ confidence interval of the values of healthy control subjects were considered significantly abnormal.

The proportion of "disconnected voxels" in each patient was defined as the ratio between the number of voxels with significantly decreased connectivity compared with healthy control subjects and the total number of voxels. The percentage of disconnected voxels was tested for associations with patient age and severity of injury using the nonparametric Spearman $\rho$ correlation coefficient. Similarly, the percentage of voxels with significantly increased connectivity relative to the controls was also calculated and tested. We compared the IC across voxels in a given subject to a null-distribution obtained from controls. Therefore, the " $\mathrm{x} \%$ of voxels" that are decreased or increased is the proportion of voxels of the entire brain that lie in the outlier of this null distribution and show a statistically significant difference from the controls. Hence, the "x\% of voxels" with altered connectivity reflects the proportion of brain affected regardless of the spatial distribution of these connectivity changes. 
Comparisons of these regions of altered functional connectivity across sequential scans were performed using the nonparametric Wilcoxon signed-rank test. False discovery rate correction of $10 \%$ was used for multiple voxel-wise comparisons. Correlation of percentage of disconnected voxels with clinical injury severity as measured by the GCS score obtained in the emergency department and with symptoms as measured by the HISC score were both made with the nonparametric Spearman $\rho$ correlation coefficient. Comparisons of the demographic characteristics between the patient and control groups were made using the Student t-test. All statistical calculations were performed using SPSS (IBM Corp.).

\section{Results}

\section{Demographics and Injury Characteristics}

In a 4-year period, 21 patients were enrolled in the study (Table 1). There were 14 male and 7 female patients, and ages ranged from 17 to 53 years, with a median age of 29 years. Nineteen patients were right-handed and 2 were left-handed. Seventeen patients had recorded loss of consciousness at the scene, 3 had no loss of consciousness and, in 1 patient, loss of consciousness status was unknown. Eighteen patients reported posttraumatic amnesia, 2 patients had no posttraumatic amnesia, and, in 1 patient, posttraumatic amnesia status was unknown. By initial GCS criteria, 11 patients had mild (GCS Score 13-15), 1 had moderate (GCS Score 9-12), and 3 had severe (GCS score < 9) TBI, and the 6 remaining patients had no initial GCS score recorded. One patient underwent a hemicraniectomy, 2 patients had external ventricular drains placed, and the remaining patients did not undergo operative intervention.

The median length of time between injury and MEG/ MRI was 14 months (range 4-27 months); for patients who underwent a second MEG, the median time to second scan was 26 months (range $21-31$ months).

On the 3-T MRI protocol (Table 2), 16 patients had abnormal findings directly attributable to TBI, 2 had only abnormal foci of T2-weighted signal hyperintensity in white matter that were nonspecific, and 3 had normal findings except for a developmental venous anomaly in 1 case. The abnormal findings specific for trauma were microbleeds in 14 cases and cortical contusions in 8 cases, with 6 patients who had both microbleeds and contusions.

Microhemorrhage and microbleed are terms commonly used in the MRI literature to refer to small hemorrhages, often less than $1 \mathrm{~mm}$ in actual size, but which are detectable by gradient echo T2*-weighted pulse sequences. These microbleeds can be observed even in the chronic phase of TBI because of residual paramagnetic blood breakdown products such as hemosiderin and ferritin, especially by high-field MRI of $3 \mathrm{~T}$ or greater.

The control group consisted of 14 men and 4 women, for a total of 18 subjects. The ages ranged from 19 to 49 years, with a median of 25 years. Seventeen control subjects were right-handed and 1 was left-handed. The control group showed no statistically significant differences from the test group regarding age, sex, or handedness ( $p>0.05)$. Three-Tesla MRI of the control subjects showed normal findings in 14 cases, nonspecific small foci of T2-weighted signal hyperintensity in white matter for 3 cases, and cerebellar tonsillar ectopia, a normal variant, in 1 case. 


\section{Results From Initial MEG Scans}

After correction for multiple voxel-wise comparisons using a $10 \%$ FDR, the percentage of disconnected voxels in the patient group had a median of $0.18 \%$ and mean of $0.5 \%$ (range $0 \%-2.85 \%$ ), while the percentage of voxels with increased connectivity after the same correction had a median of $0 \%$ and a mean of $0.27 \%$ (range $0 \%-3.85 \%$ ). As a group, the TBI patients demonstrated a significant decrease in functional connectivity compared with the control group $(\mathrm{p}<0.01)$ (Fig. 1). There was a weakly positive association between the percentage of disconnected voxels and HISC scores $(\rho=0.35, p=0.18)$, where higher scores on the HISC questionnaire signify a greater number of posttraumatic symptoms, although this trend did not reach statistical significance. There was no significant correlation between the percentage of disconnected voxels and GCS score assessed in the emergency department $(\rho=0.22, p=0.44)$. There was also no significant correlation between the percentage of disconnected voxels and time interval between the injury and the MEG scan ( $\rho$ $=0.30, \mathrm{p}=0.24)$.

Of the 5 TBI patients with normal findings on 3-T MRI, or nonspecific findings not likely to be related to trauma, 3 had regions of disconnected voxels by MEG and the other 2 had no disconnected voxels.

\section{Results From Follow-Up MEG Scans}

Five of the TBI patients underwent a second MEG scan approximately 2 years after the first scan. At a 10\% FDR correction for multiple comparisons, the MEG scans at the second time point demonstrated a significantly lower percentage of voxels with decreased connectivity ( $p$ $<0.05)$ and a trend toward a higher percentage of voxels with increased connectivity ( $p=$ 0.18) compared with the initial MEG (Table 3). Because of the small number of patients who underwent follow-up scanning, a group analysis of follow-up versus baseline scans is not adequately powered for definitive conclusions. Instead, to illustrate interesting findings on a case-by-case basis, 3 patients with TBI were each compared across their own serial scans, as discussed below in greater detail.

\section{Case 2: Mild TBI From Bicycle Accident}

This 28-year-old right-handed man with no prior medical history was hit by an opening car door while he was riding his bicycle. The patient was helmeted; he lost consciousness for approximately 1 minute and displayed posttraumatic amnesia. His GCS score in the field was 14 (M6V4E4), which improved to 15 upon evaluation in the emergency department. His initial CT scan was interpreted by a board-certified neuroradiologist as negative for head injury. He was diagnosed in the emergency department with concussive head injury, and he was subsequently discharged.

In the following 3 months, the patient experienced significant symptoms of intermittent headache, nausea, and increased fatigue. These symptoms largely resolved at 6 months; however, he did have persistent difficulty with concentration and multitasking. His partner also reported that the patient exhibited increased mood swings. Two years after his injury, the patient reported near complete resolution of his symptoms and reported that his quality of life was back to his preinjury baseline. 
Three-Tesla MRI, performed 9 months after injury using the research protocol described in Methods showed numerous microbleeds in the high right frontal lobe white matter as well as 1 microbleed at the posterior left frontal lobe gray-white matter junction, all consistent with hemorrhagic axonal shear injury.

The patient's initial MEG scan was obtained 9 months after his injury (Fig. 2 left). It demonstrated several regions of decreased resting state functional connectivity compared with the control group, particularly in the bilateral parietooccipital regions and the right frontoparietal region. After multiple comparisons correction using a 10\% FDR, 1.5\% of voxels had significantly less connectivity than the control group. No voxels were found to have increased connectivity compared with the control group.

The second MEG scan was obtained 23 months after the initial scan (Fig. 2 right). Overall, it demonstrated a decrease in the volume of cortex with reduced connectivity, although some of the cortical regions with the greatest reductions in functional connectivity on the initial MEG remained abnormal on the follow-up MEG. Quantitatively, after 10\% FDR correction, there were $0.65 \%$ of voxels with persistently reduced connectivity, while $0.04 \%$ of voxels now displayed increased connectivity compared with the control group. Overall, this change in functional connectivity over the 23 -month period is statistically significant $(\mathrm{p}<0.01)$.

\section{Case 12: Mild TBI After Rollover Motor Vehicle Accident}

This 17-year-old right-handed male patient was a restrained back-seat passenger in a highspeed rollover motor vehicle accident with an extraction time of almost 1 hour. Although he had an initial loss of consciousness of 15 minutes, the patient's GCS score was 15 at the time of initial examination (after extraction), and remained 15 in the emergency department. He exhibited posttraumatic amnesia, but of less than 24 hours' duration. He was observed overnight in the ICU and then discharged from the hospital on Day 3.

In the following months, the patient had some headaches and difficulty with concentration. These symptoms cleared quite rapidly, however, and, by the time of his 2-year follow-up MEG scan, his HISC score was 1 . He has since graduated from college.

The patient's initial CT scan demonstrated multiple, bilateral microbleeds in the deep white matter consistent with hemorrhagic axonal shear injury. A 3-T MR image obtained 8 days later under the research protocol confirmed numerous bilateral microbleeds, especially in the left frontal and temporal lobes.

The patient's first MEG scan was obtained 1 month after the injury (Fig. 3 left). It demonstrated small patches of decreased connectivity in the right cerebral hemisphere. After correction for $10 \%$ FDR, connectivity analysis demonstrates $0.18 \%$ of voxels having significantly less connectivity than the control group; $0.044 \%$ of voxels were found to have increased connectivity compared with control group.

The subsequent MEG study was performed 26 months later, and it showed a dramatic change from the original scan (Fig. 3 right). No voxels exhibited decreased functional connectivity, and $3.5 \%$ of voxels demonstrated increased connectivity as compared with 
controls. The areas of increased connectivity are predominantly localized to the bilateral parietal and left temporal regions.

\section{Case 5: Severe TBI After Bicycle Striking an Automobile}

This 44-year-old right-handed helmeted bicyclist collided with an automobile. His initial GCS score in the field was 7 (M5V1E1) with loss of consciousness and posttraumatic amnesia. His GCS score in the emergency department increased to 8 (M4V2E2). Although he did not undergo surgery, an external ventricular drain and brain tissue oxygen monitor were placed. The patient remained in the ICU for 1 week postoperatively and was discharged from the hospital to rehabilitation.

In the following months, the patient did have typical symptoms following head injury, including headaches, nausea, and difficulty with concentration. His HISC score was 7. At the 2-year follow-up appointment, he reported continuing symptoms including headache and difficulty with concentration that had not improved. Although a follow-up HISC score was unavailable, the patient reported feeling "about the same" as he had a month after the injury.

The patient's initial CT scan demonstrated bifrontal subcortical microbleeds, right frontal and temporooccipital contusions, and a right perisylvian subarachnoid hemorrhage. A 3-T MR image obtained 7 months later under the research protocol demonstrated innumerable microbleeds throughout both hemispheres including the corpus callosum as well as chronic hemorrhagic right frontal, occipital, and temporal contusions.

The patient's first MEG scan was completed 11 months after the injury (Fig. 4A). It demonstrated small areas of decreased connectivity, predominantly in the bilateral parietooccipital regions compared with the control group. After correction with $10 \%$ FDR, $0.040 \%$ of voxels had significantly decreased connectivity compared with the control group, but no voxels had increased connectivity. His second MEG scan was completed 31 months after the initial scan (Fig. 4B). It demonstrated a similar connectivity pattern with $0.036 \%$ of voxels demonstrating significantly decreased connectivity compared with controls. Interestingly, although he did demonstrate some evidence of cortical improvement, worsening connectivity was observed in the right cerebellum.

\section{Other Cases With Serial MEG Scans}

The 2 other patients (Cases 3 and 7) with follow-up MEG scans also did not demonstrate the recovery of resting state functional connectivity observed in the patients in Cases 2 and 12, but rather showed persistently reduced connectivity in the same regions as the initial scan (Fig. 4C and D for Case 7 and Fig. 4E and F for Case 3).

\section{Discussion}

Traumatic brain injury, even when considered mild by GCS criteria, often results in longterm cognitive and behavioral impairment. The degree of recovery experienced by patients who have suffered head trauma cannot be reliably predicted from demographic characteristics, standard clinical measures of injury severity, or conventional neuroimaging, especially for mild TBI, which is often described as "concussion." A better understanding of 
the pathophysiological processes in TBI is needed to develop biomarkers that are effective in prognosticating outcome and predicting response to interventions such as pharmacotherapy or cognitive rehabilitation. In this pilot investigation of resting-state MEG in TBI, we found supporting evidence for our hypothesis that disruption of the long-range communication between brain regions, as measured by reductions in functional connectivity, would be found in patients compared with matched controls. Magnetoencephalography also detected abnormally low functional connectivity in 3 of 5 patients without radiographically evident traumatic lesions on 3-T MR imaging, suggesting a potentially high degree of sensitivity to brain injury. Furthermore, we observed in 2 cases that long-term recovery from the effects of TBI was accompanied by improvements in these areas of disrupted connectivity on follow-up MEG scans and, in 1 striking case, by the development of new areas of elevated functional connectivity compared with controls.

Prior MEG studies of TBI have reported increased slow waves in localized brain regions that correlate with patient symptoms. ${ }^{16,22}$ The more recent study by Huang et al. ${ }^{16}$ revealed slow waves in TBI patients with normal MRI findings and even with normal DTI findings, suggesting that MEG may be more sensitive to the deleterious effects of head injury than these MRI-based technologies. This result is in accord with our finding of abnormal restingstate MEG functional connectivity in 3 of 5 patients with either normal anatomical findings or nonspecific foci of white matter T2-weighted hyperintensity that were also seen in some of our control subjects and are commonly found in asymptomatic healthy individuals using modern high-resolution MRI at $3 \mathrm{~T} .{ }^{16}$ However, to our knowledge, there has been only one prior investigation that directly examined resting-state MEG functional connectivity in brain trauma. Castellanos et al. ${ }^{3}$ found reduced alpha band functional connectivity in patients with severe TBI compared with normal controls, a deficit that diminished over time after administration of cognitive rehabilitation. These results agree with our finding of decreased functional connectivity at alpha frequencies that improve upon recovery; however, we extend this observation to show that this phenomenon can be observed in mild TBI as well. The prior MEG study was also limited by poorer spatial resolution that only allowed localization of connectivity at lobar spatial scales, due to fewer MEG sensors, older source reconstruction methods, and a measure of connectivity that did not account for volume conduction effects. ${ }^{3}$ Our results show that abnormal MEG functional connectivity can be localized to within $1 \mathrm{~cm}$ using short recording periods of 1 minute or less that can be easily performed in the clinical setting. Another advantage of our technique is that regions of pathological connectivity can be rapidly determined in each patient by comparison with a control MEG database using fully automated methods, thereby eliminating subjective observer bias and interrater variability.

Interestingly, the magnitude of the disruption as measured by the percentage of voxels with decreased connectivity did not correlate with the severity of injury (evaluated by the GCS), with symptoms (measured by the HISC), or with the duration of time since the traumatic event, although a positive trend was seen between more functional disconnection and a greater number of post-traumatic symptoms. This pilot study is probably underpowered to detect these effects; hence, a larger series may be necessary with correlation to neurocognitive outcome, which can be measured more objectively than symptoms. However, our findings are consistent with prior studies ${ }^{20,27,39}$ and do illustrate an important 
characteristic of TBI — that even in the absence of a focal lesion evident on conventional neuroimaging, TBI can disrupt connectivity in widely disparate brain regions. Additionally, regions of connectivity disruption did not necessarily correspond to the locations of focal lesions on 3-T MR scans; in short, connectivity disruption often occurs at locations remote from the sites of radiographic injury. This study also reports the results of follow-up MEG scans performed in 5 patients in the cohort (Figs. 2-4). To our knowledge, this is the first reported series of sequential MEG scans in patients who have suffered TBI. Although the patients in this subgroup are heterogeneous both in demographics and in clinical course, analysis of these follow-up scans yields a valuable finding: over a period of time (in this case, approximately 2 years), the connectivity deficits can improve in some cases. Indeed, even with this relatively small subgroup, there are statistically significant levels of improvement in disconnected voxels. Furthermore, the trend toward an increase in "overconnected" regions during this time period suggests that other brain regions might be compensating for the injured regions. These findings are consistent with prior reports of reorganization in neural networks after brain injury, a phenomenon known as neuroplasticity. $3,17,27$ The patient in Case 12 who showed strikingly increased functional connectivity on the follow-up MEG (Fig. 3) was the youngest in our cohort (17 years old) and therefore possibly capable of a greater degree of neuroplasticity in response to injury.

One limitation of this study is the heterogeneity of clinical presentations among patients. While the majority of patients suffered from mild TBI and underwent no operative intervention, there were cases of moderate and severe TBI, and 3 patients underwent operative procedures. Additionally, due to the difficulties in patient recruitment and scheduling, the time period between injury and MEG scan varied across the group. Admittedly, having more homogeneity in both these factors would be ideal. However, the fact that statistically significant reductions in connectivity are detectable despite these factors lends robustness to the conclusions.

Another factor that must be addressed is the inter- and intrasession test-retest reliability. A prior study from our group examined global resting-state MEG connectivity maps by calculating a fixed-effects average ICC. This study showed good test-retest reliability for both within-session $(\mathrm{ICC}=0.61)$ and intersession $(\mathrm{ICC}=0.64)$ data. ${ }^{15}$ These values compare favorably with those found in studies of test-retest reliability in resting state functional MRI. ${ }^{13,37,40}$ Also, the similarities over most of the brain between the baseline and 2-year interval scans in the subgroup of 5 patients lend further credence to the consistency of these connectivity maps.

Larger studies are needed to gather enough connectivity data to construct a comprehensive model of how various mechanisms and severities of TBI affect resting state functional connectivity. Investigation of other MEG frequency ranges besides the alpha band might also provide more information regarding functional disconnectivity; indeed, Castellanos et al. ${ }^{3}$ reported increases in delta band connectivity due to TBI that decrease with rehabilitation, the opposite of what they and we observed for alpha waves. It should be noted that this pilot study is not intended to compare MEG with MRI as an indicator of functional outcome; instead, our pilot results suggest the potential for MEG functional connectivity to serve as a sensitive biomarker for traumatic brain dysfunction. While significant changes in 
IC were found between TBI patients and controls, the degree of change in IC within a specific voxel indicates a reduction in non-phase-locked correlations between that particular voxel and the rest of the brain. We report only regions in which IC differences between patients and controls were statistically significant. As for the functional significance of this finding, it remains to be established further. So far, we can only establish the phenomenology and we cannot yet elucidate its neural mechanisms.

Future large-scale studies are needed to test this potential and to examine the utility of MEG as a prognostic and predictive TBI biomarker for use in patient counseling, triage to clinical research studies, and monitoring the effectiveness of interventions.

\section{Conclusions}

This pilot investigation of a rapid automated resting-state MEG imaging technique suitable for routine clinical application in TBI demonstrates abnormally decreased functional connectivity between brain regions that may persist for years after the injury, including cases classified as "mild" by GCS criteria. Regions of altered connectivity do not necessarily correlate with focal traumatic lesions on 3-T MR scans, and abnormal MEG connectivity can be detected even in some patients with normal 3-T MRI. Analysis of follow-up MEG scans in a subgroup of patients shows that, over time, the abnormally reduced connectivity can improve, suggesting neuroplasticity during the recovery from TBI. Resting-state MEG deserves further investigation as a prognostic and predictive biomarker for TBI.

\section{Acknowledgments}

This work was supported by the Dana Foundation Brain and Immuno-imaging Program, UCSF Research Evaluation and Allocation Program, and NIH (Grant Nos. R01 NS060776 and RC2 NS069409).

\section{Abbreviations used in this paper}

$\begin{array}{ll}\text { FDR } & \text { false discovery rate } \\ \text { GCS } & \text { Glasgow Coma Scale } \\ \text { HISC } & \text { Head Injury Symptom Checklist } \\ \text { IC } & \text { imaginary coherence } \\ \text { ICC } & \text { intraclass correlation coefficient } \\ \text { MEG } & \text { magnetoencephalography } \\ \text { TBI } & \text { traumatic brain injury }\end{array}$

\section{References}

1. Aertsen AM, Gerstein GL, Habib MK, Palm G. Dynamics of neuronal firing correlation: modulation of "effective connectivity". J Neurophysiol. 1989; 61:900-917. [PubMed: 2723733]

2. Bartolomei F, Bosma I, Klein M, Baayen JC, Reijneveld JC, Postma TJ, et al. How do brain tumors alter functional connectivity? A magnetoencephalography study. Ann Neurol. 2006; 59:128-138. [PubMed: 16278872] 
3. Castellanos NP, Paul N, Ordonez VE, Demuynck O, Bajo R, Campo P, et al. Reorganization of functional connectivity as a correlate of cognitive recovery in acquired brain injury. Brain. 2010; 133:2365-2381. [PubMed: 20826433]

4. da Silva FH, van Lierop TH, Schrijer CF, van Leeuwen WS. Organization of thalamic and cortical alpha rhythms: spectra and coherences. Electroencephalogr Clin Neurophysiol. 1973; 35:627-639. [PubMed: 4128158]

5. Dalal SS, Zumer JM, Guggisberg AG, Trumpis M, Wong DD, Sekihara K, et al. MEG/EEG source reconstruction, statistical evaluation, and visualization with NUTMEG. Comput Intell Neurosci. 2011; 2011:758973. [PubMed: 21437174]

6. Douw L, Baayen H, Bosma I, Klein M, Vandertop P, Heimans J, et al. Treatment-related changes in functional connectivity in brain tumor patients: a magnetoencephalography study. Exp Neurol. 2008; 212:285-290. [PubMed: 18534578]

7. Eliassen JC, Boespflug EL, Lamy M, Allendorfer J, Chu WJ, Szaflarski JP. Brain-mapping techniques for evaluating poststroke recovery and rehabilitation: a review. Top Stroke Rehabil. 2008; 15:427-450. [PubMed: 19008203]

8. Faul, M.; Xu, L.; Wald, MM.; Coronado, VG. Traumatic Brain Injury in the United States: Emergency Department Visits, Hospitalizations, and Deaths. Atlanta: National Center for Injury Prevention and Control, Centers for Disease Control and Prevention; 2010. (http://www.cdc.gov/ traumaticbraininjury/pdf/blue_book.pdf) [Accessed March 14, 2013]

9. Fein G, Raz J, Brown FF, Merrin EL. Common reference coherence data are confounded by power and phase effects. Electroencephalogr Clin Neurophysiol. 1988; 69:581-584. [PubMed: 2453336]

10. Finkelstein, EA.; Corso, PS.; Miller, TR. The Incidence and Economic Burden of Injuries in the United States. New York: Oxford University Press; 2006.

11. Friston KJ, Frith CD, Liddle PF, Frackowiak RS. Functional connectivity: the principal-component analysis of large (PET) data sets. J Cereb Blood Flow Metab. 1993; 13:5-14. [PubMed: 8417010]

12. Guggisberg AG, Honma SM, Findlay AM, Dalal SS, Kirsch HE, Berger MS, et al. Mapping functional connectivity in patients with brain lesions. Ann Neurol. 2008; 63:193-203. [PubMed: 17894381]

13. Guo CC, Kurth F, Zhou J, Mayer EA, Eickhoff SB, Kramer JH, et al. One-year test-retest reliability of intrinsic connectivity network fMRI in older adults. Neuroimage. 2012; 61:14711483. [PubMed: 22446491]

14. Hinkley LB, Owen JP, Fisher M, Findlay AM, Vinogradov S, Nagarajan SS. Cognitive impairments in schizophrenia as assessed through activation and connectivity measures of magnetoencephalography (MEG) data. Front Hum Neurosci. 2010; 3:73. [PubMed: 21160543]

15. Hinkley LB, Vinogradov S, Guggisberg AG, Fisher M, Findlay AM, Nagarajan SS. Clinical symptoms and alpha band resting-state functional connectivity imaging in patients with schizophrenia: implications for novel approaches to treatment. Biol Psychiatry. 2011; 70:11341142. [PubMed: 21861988]

16. Huang MX, Theilmann RJ, Robb A, Angeles A, Nichols S, Drake A, et al. Integrated imaging approach with MEG and DTI to detect mild traumatic brain injury in military and civilian patients. J Neurotrauma. 2009; 26:1213-1226. [PubMed: 19385722]

17. Jenkins WM, Merzenich MM. Reorganization of neocortical representations after brain injury: a neurophysiological model of the bases of recovery from stroke. Prog Brain Res. 1987; 71:249266. [PubMed: 3588947]

18. Lachaux JP, Rodriguez E, Martinerie J, Varela FJ. Measuring phase synchrony in brain signals. Hum Brain Mapp. 1999; 8:194-208. [PubMed: 10619414]

19. Le Van Quyen M, Foucher J, Lachaux J, Rodriguez E, Lutz A, Martinerie J, et al. Comparison of Hilbert transform and wavelet methods for the analysis of neuronal synchrony. J Neurosci Methods. 2001; 111:83-98. [PubMed: 11595276]

20. Lee RG, van Donkelaar P. Mechanisms underlying functional recovery following stroke. Can J Neurol Sci. 1995; 22:257-263. [PubMed: 8599767]

21. Lewine JD, Davis JT, Bigler ED, Thoma R, Hill D, Funke M, et al. Objective documentation of traumatic brain injury subsequent to mild head trauma: multimodal brain imaging with MEG, SPECT, and MRI. J Head Trauma Rehabil. 2007; 22:141-155. [PubMed: 17510590] 
22. Lewine JD, Davis JT, Sloan JH, Kodituwakku PW, Orrison WW Jr. Neuromagnetic assessment of pathophysiologic brain activity induced by minor head trauma. AJNR Am J Neuroradiol. 1999; 20:857-866. [PubMed: 10369357]

23. Martino J, Honma SM, Findlay AM, Guggisberg AG, Owen JP, Kirsch HE, et al. Resting functional connectivity in patients with brain tumors in eloquent areas. Ann Neurol. 2011; 69:521532. [PubMed: 21400562]

24. Nakamura T, Hillary FG, Biswal BB. Resting network plasticity following brain injury. PLoS ONE. 2009; 4:e8220. [PubMed: 20011533]

25. National Center for Injury Prevention and Control. Report to Congress on Mild Traumatic Brain Injury in the United States: Steps to Prevent a Serious Public Health Problem. Atlanta: Centers for Disease Control and Prevention; 2003. (http://www.cdc.gov/ncipc/pub-res/mtbi/mtbireport.pdf) [Accessed March 14, 2013]

26. Nolte G, Bai O, Wheaton L, Mari Z, Vorbach S, Hallett M. Identifying true brain interaction from EEG data using the imaginary part of coherency. Clin Neurophysiol. 2004; 115:2292-2307. [PubMed: 15351371]

27. Nudo RJ, Wise BM, SiFuentes F, Milliken GW. Neural substrates for the effects of rehabilitative training on motor recovery after ischemic infarct. Science. 1996; 272:1791-1794. [PubMed: 8650578]

28. Nunez PL, Srinivasan R, Westdorp AF, Wijesinghe RS, Tucker DM, Silberstein RB, et al. EEG coherency. I: Statistics, reference electrode, volume conduction, Laplacians, cortical imaging, and interpretation at multiple scales. Electroencephalogr Clin Neurophysiol. 1997; 103:499-515. [PubMed: 9402881]

29. Quigley M, Cordes D, Wendt G, Turski P, Moritz C, Haughton V, et al. Effect of focal and nonfocal cerebral lesions on functional connectivity studied with MR imaging. AJNR Am J Neuroradiol. 2001; 22:294-300. [PubMed: 11156772]

30. Salvador R, Suckling J, Coleman MR, Pickard JD, Menon D, Bullmore E. Neurophysiological architecture of functional magnetic resonance images of human brain. Cereb Cortex. 2005; 15:1332-1342. [PubMed: 15635061]

31. Sekihara K, Nagarajan SS, Poeppel D, Marantz A. Asymptotic SNR of scalar and vector minimum-variance beamformers for neuromagnetic source reconstruction. IEEE Trans Biomed Eng. 2004; 51:1726-1734. [PubMed: 15490820]

32. Sekihara K, Nagarajan SS, Poeppel D, Marantz A, Miyashita Y. Reconstructing spatio-temporal activities of neural sources using an MEG vector beamformer technique. IEEE Trans Biomed Eng. 2001; 48:760-771. [PubMed: 11442288]

33. Sigurdardottir S, Andelic N, Roe C, Jerstad T, Schanke AK. Post-concussion symptoms after traumatic brain injury at 3 and 12 months post-injury: a prospective study. Brain Inj. 2009; 23:489-497. [PubMed: 19484622]

34. Stam CJ, Breakspear M, van Cappellen van Walsum AM, van Dijk BW. Nonlinear synchronization in EEG and whole-head MEG recordings of healthy subjects. Hum Brain Mapp. 2003; 19:63-78. [PubMed: 12768531]

35. Stam CJ, van Cappellen van Walsum AM, Pijnenburg YA, Berendse HW, de Munck JC, Scheltens $\mathrm{P}$, et al. Generalized synchronization of MEG recordings in Alzheimer's Disease: evidence for involvement of the gamma band. J Clin Neurophysiol. 2002; 19:562-574. [PubMed: 12488788]

36. Vrba J, Robinson SE. Signal processing in magnetoencephalography. Methods. 2001; 25:249-271. [PubMed: 11812209]

37. Wang JH, Zuo XN, Gohel S, Milham MP, Biswal BB, He Y. Graph theoretical analysis of functional brain networks: test-retest evaluation on short- and long-term resting-state functional MRI data. PLoS ONE. 2011; 6:e21976. [PubMed: 21818285]

38. Westlake KP, Nagarajan SS. Functional connectivity in relation to motor performance and recovery after stroke. Front Syst Neurosci. 2011; 5:8. [PubMed: 21441991]

39. Witte OW, Stoll G. Delayed and remote effects of focal cortical infarctions: secondary damage and reactive plasticity. Adv Neurol. 1997; 73:207-227. [PubMed: 8959216] 
40. Zuo XN, Kelly C, Adelstein JS, Klein DF, Castellanos FX, Milham MP. Reliable intrinsic connectivity networks: test-retest evaluation using ICA and dual regression approach. Neuroimage. 2010; 49:2163-2177. [PubMed: 19896537] 


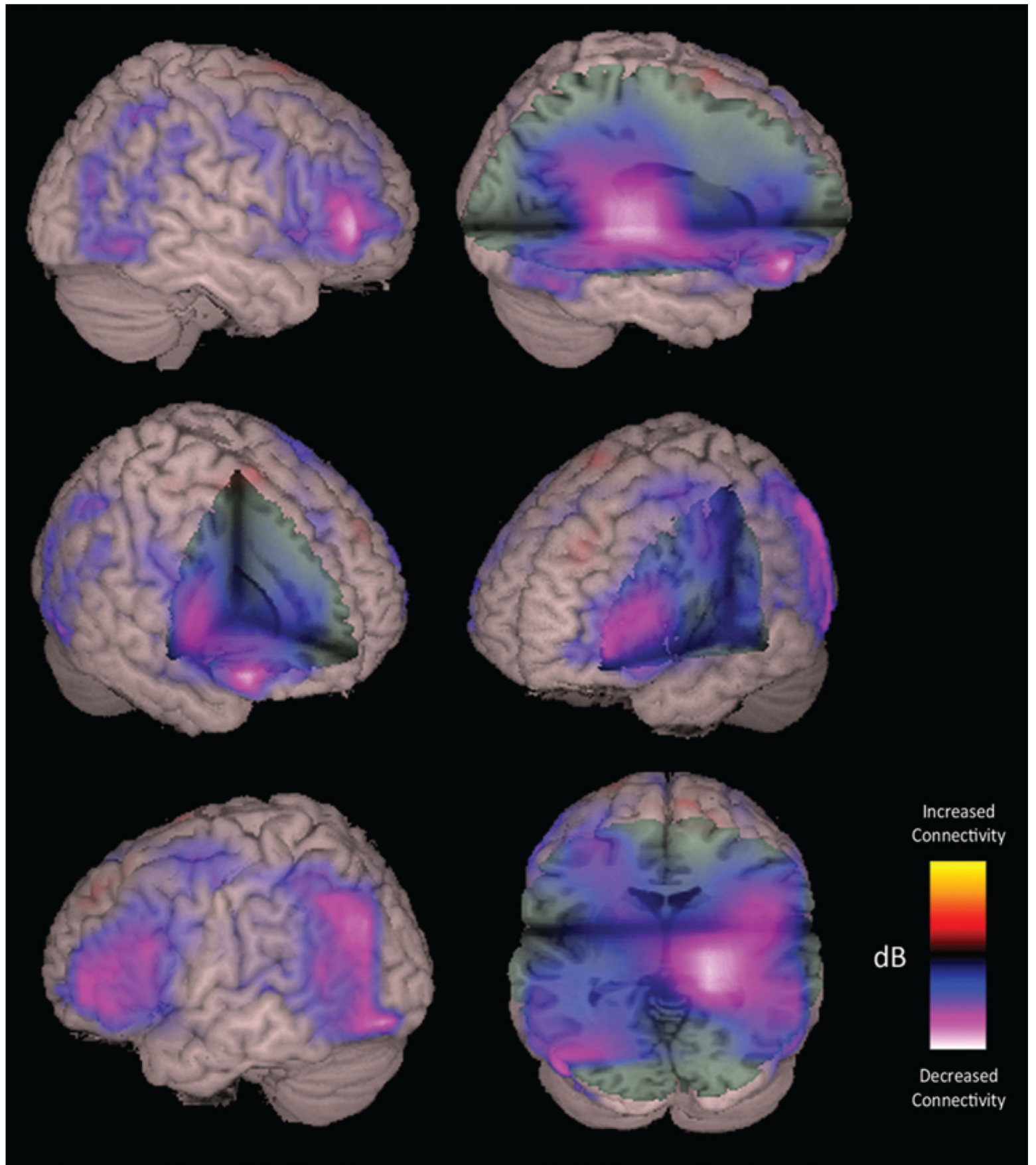

Fig. 1.

Group comparison of 21 TBI patients versus 18 controls. Three-dimensional volumetric renderings of the MEG results coregistered to the T1-weighted structural MRI show significant reduction in functional connectivity of the patients, predominantly in the bilateral frontal and left greater than right parietotemporooccipital regions as well as the right thalamus. Increased connectivity is represented in the red $\rightarrow$ orange spectrum; decreased connectivity is depicted in the blue $\rightarrow$ white spectrum. 

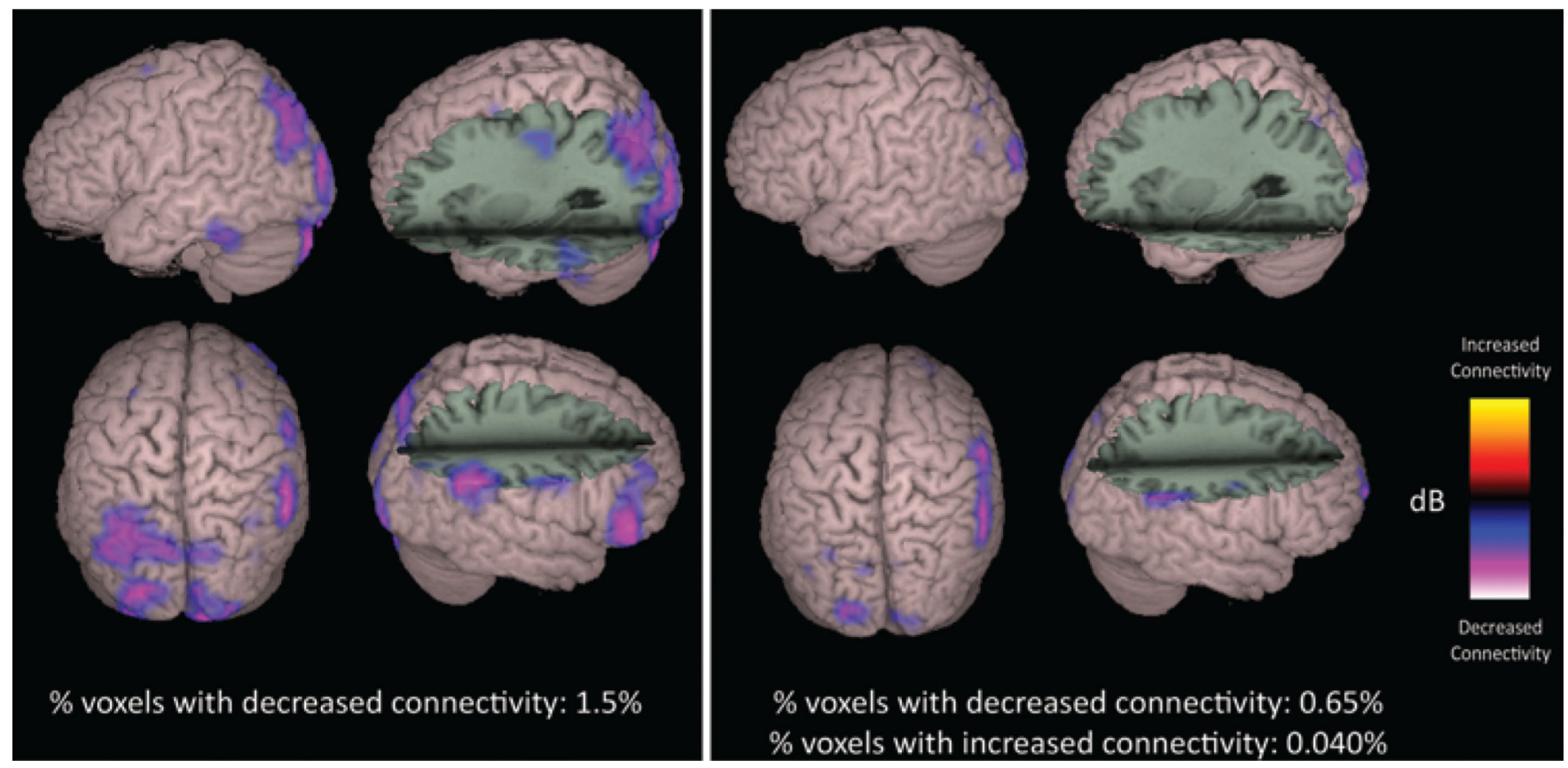

Fig. 2.

Case 2. Three-dimensional renderings of the initial (left) and 23-month follow-up (right) MEG studies. Percentages of voxels with significantly increased and/or decreased connectivity compared with controls are listed below the montage. Image display conventions are as in Fig. 1. 

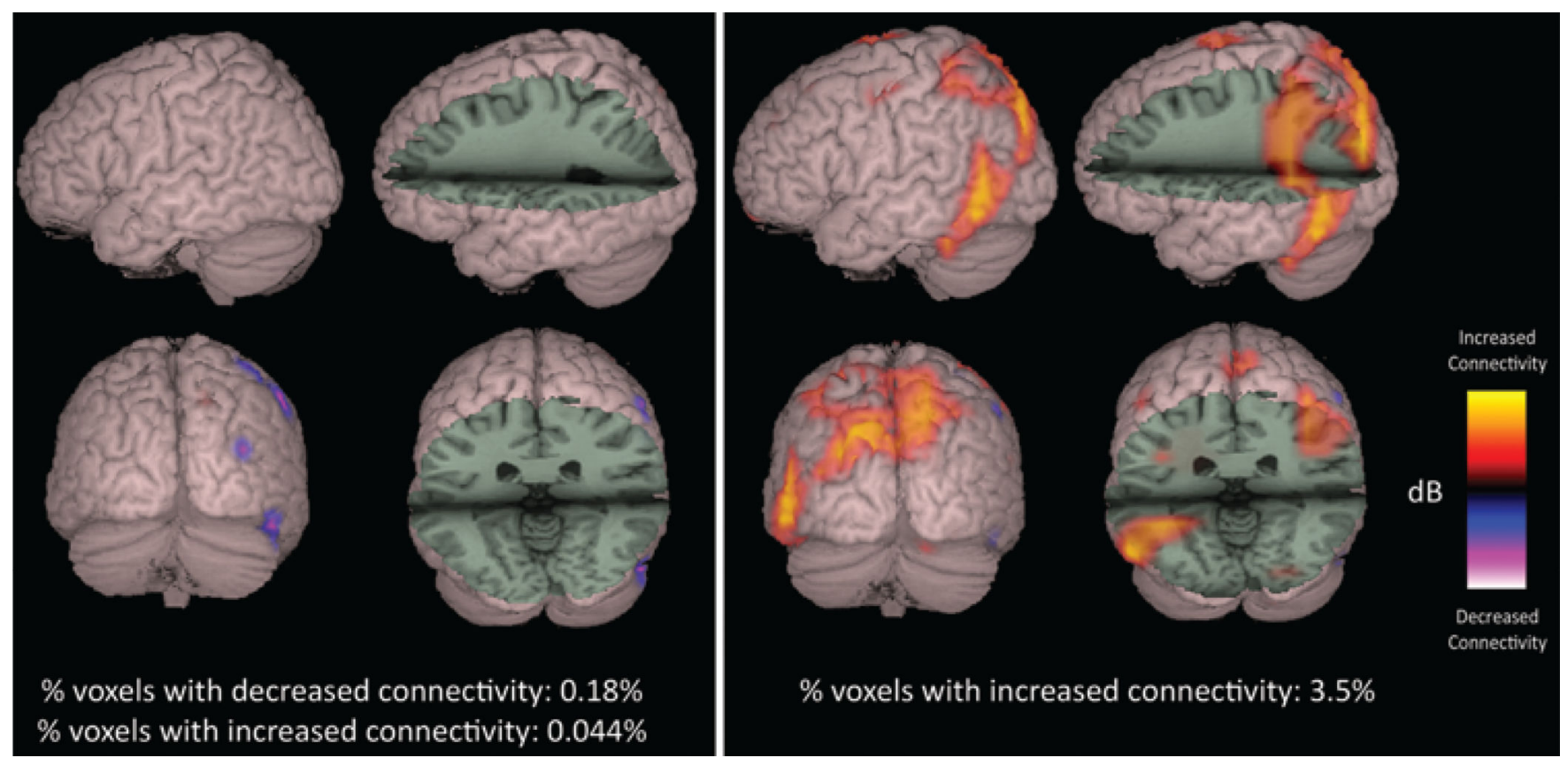

Fig. 3.

Case 12. Three-dimensional renderings of the initial (left) and 26-month follow-up (right)

MEG studies. Percentages of voxels with significantly increased and/or decreased connectivity compared with controls are listed below the montage. Image display conventions are as in Fig. 1. 


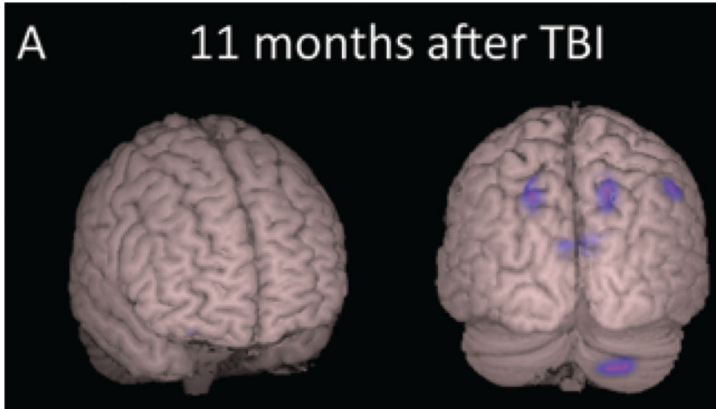

$\%$ voxels with decreased connectivity: $0.040 \%$
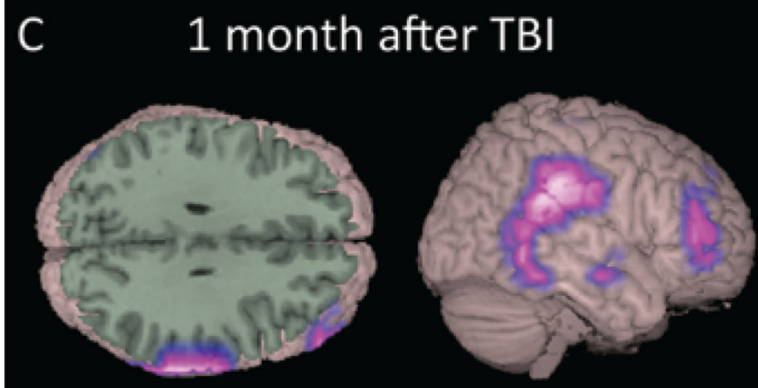

$\%$ voxels with decreased connectivity: $0.47 \%$

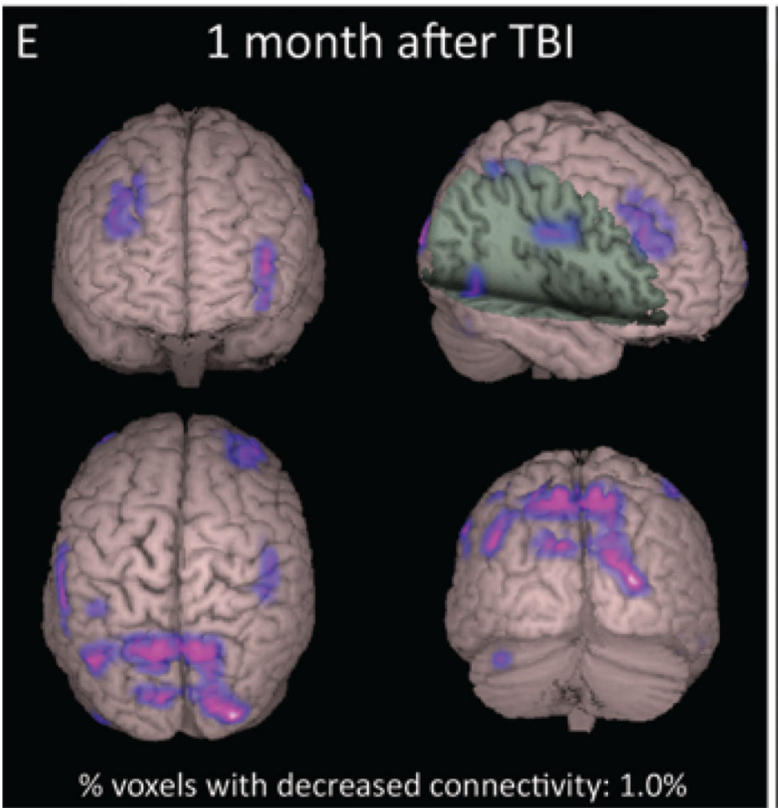

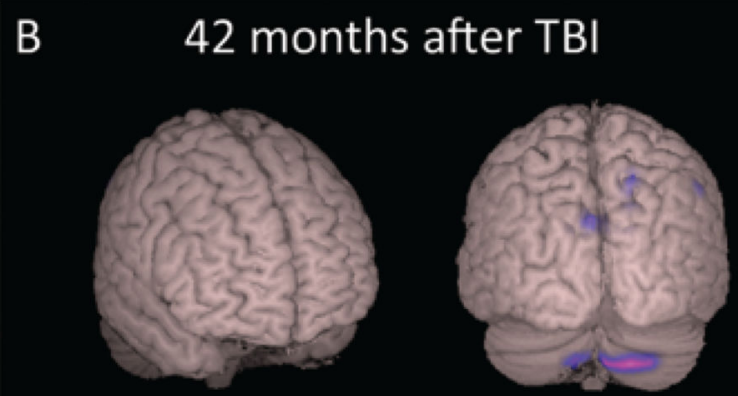

$\%$ voxels with decreased connectivity: $0.036 \%$

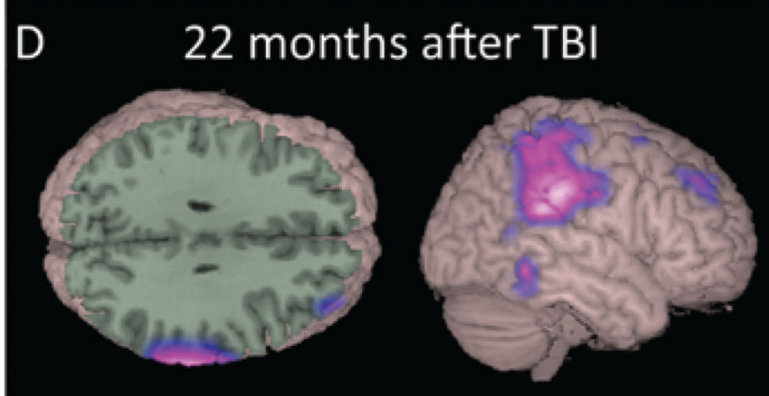

$\%$ voxels with decreased connectivity: $0.44 \%$

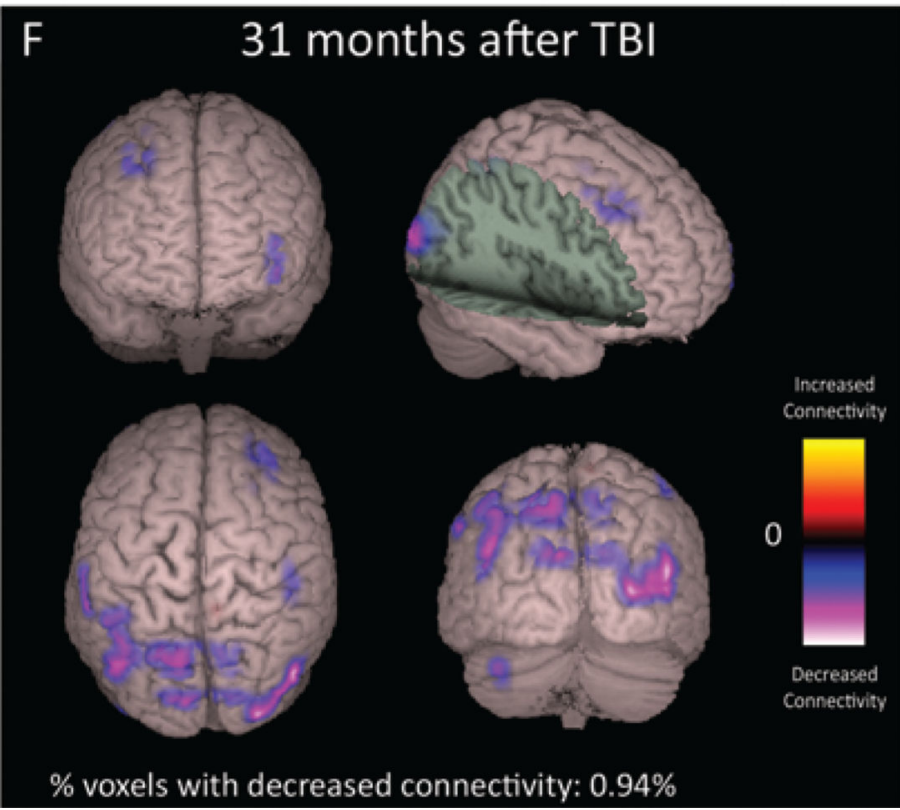

Fig. 4.

Case 5. Three-dimensional renderings of the initial (A) and 31-month follow-up (B) MEG studies. C-F: Cases 7 and 3. Three-dimensional renderings of the initial and follow-up (at 21 and 30 months apart, respectively) MEG scans. Percentages of voxels with significantly increased and/or decreased connectivity compared with controls are listed below the montage. Image display conventions are as in Fig. 1. 


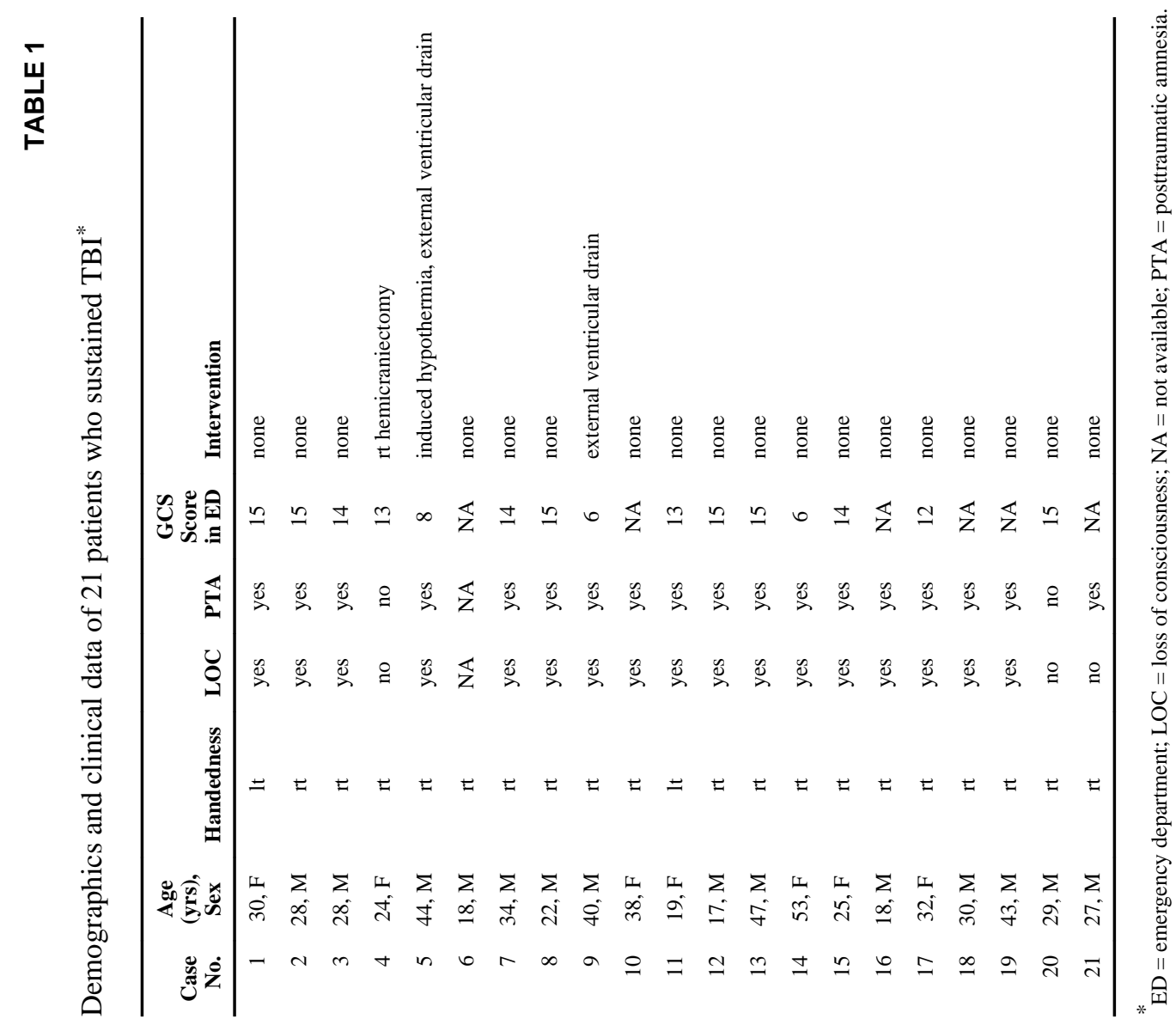

J Neurosurg. Author manuscript; available in PMC 2014 June 26. 
TABLE 2

\section{Three-Tesla MRI results in patients who sustained TBI}

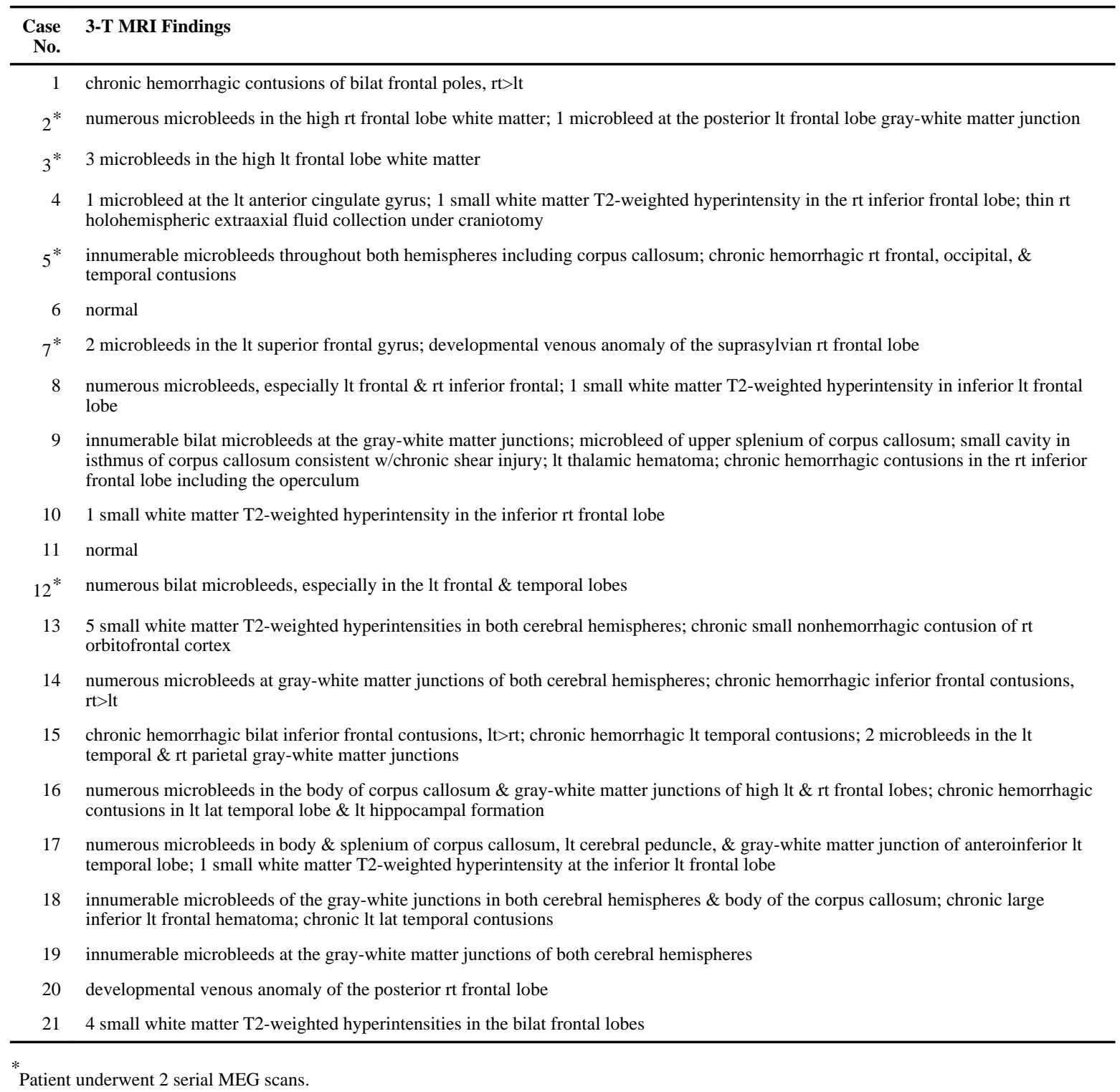




\section{TABLE 3}

Functional connectivity of 5 TBI patients with initial and follow-up MEG scans obtained approximately 2 years apart*

\begin{tabular}{clllll}
\hline & \multicolumn{4}{c}{ \% Voxels } \\
\cline { 2 - 3 } \cline { 5 - 6 } Case No. & $\downarrow$ & $\uparrow$ & & \multicolumn{2}{c}{ Visit 2 } \\
\hline 2 & 1.48 & 0.00 & 0.65 & $\uparrow$ \\
3 & 1.0 & 0.0 & 0.94 & 0.04 \\
5 & 0.04 & 0.00 & 0.036 & 0.00 \\
7 & 0.47 & 0.00 & 0.44 & 0.00 \\
12 & 0.18 & 0.044 & 0.0 & 3.5 \\
mean $\pm \mathrm{SD}$ & $0.64 \pm 0.60$ & $0.0088 \pm 0.20$ & $0.41 \pm 0.40^{\dagger}$ & $0.70 \pm 1.55^{\ddagger}$ \\
\hline
\end{tabular}

* Values indicate the percentage of voxels with decreased (down arrow) or increased (up arrow) functional connectivity. All values corrected for multiple voxel-wise comparisons using a 10\% FDR.

${ }^{\dagger} \mathrm{p}<0.05$ compared with prior MEG scan.

${ }_{\mathrm{p}}=0.18$ compared with prior MEG scan. 